\title{
A NOTE ON AN INEQUALITY FOR THE GAMMA FUNCTION
}

\author{
CHRISTOPHER OLUTUNDE IMORU \\ Department of Mathematics \\ University of Ife \\ Ile-Ife, Oyo State, Nigeria
}

(Recelved August 23, 1976 and in revised form June 30, 1977)

ABSTRACT. Some Inequalities for the Wallis functions are proved. The results of this paper are consequences of some characterization of convex functions. A generalization of a result of Boyd (1) and an extention of an inequality of Gantschi (3) are obtained.

KEY WORDS AND PHRASES. Gamma functions, characterization of convex functions, Inequalities for Gamma functions.

AMS(MOS) SUBJECT CLASSIFICATION 11970) CODES. $26 A 51,33 A 15$.

The aim of this note is to show that some inequalities for the Wallis function

$$
W(\xi, \theta)=\frac{\Gamma(\xi+1)}{\Gamma(\xi+\theta)}, \quad(\xi, \theta) \varepsilon R_{+} \times(0,1)
$$

are natural consequences of the property of convex functions or of differentiable functions. Indeed, our results are, to some extent, consequences 
of the following characterization of convex functions.

THEOREM 1. A real-valued function $\phi$ is convex on a closed interval $\bar{I} \subseteq R$ if and only if for every point $x_{0} \varepsilon \bar{I}$, the function

$$
x \longrightarrow \frac{\phi(x)-\phi\left(x_{0}\right)}{x-x_{0}}, \quad x \in \bar{I},
$$

is non-decreasing on $\bar{I}$. In particular, if $\phi$ is convex on $\bar{I}, u \neq v, x \neq y$, $u \leq x, v \leq y$, for all $u, v, x, y \in \bar{I}$, then

$$
\frac{\phi(\mathbf{v})-\phi(u)}{\mathbf{v}-\mathbf{u}} \leq \frac{\phi(y)-\phi(\mathbf{x})}{\mathbf{y}-\mathbf{x}}
$$

The proof of the theorem is well known; see for example, ([3], pp. 15-18). It is, therefore, omitted.

THEOREM 2. Let $u, v, x, y w$ and $z$ be positive real-numbers satisfying $u \neq v, w \neq z, u \leq x \leq w, x<y \leq z$ and $v \leq y$.

Then the following inequality is valid

$$
\left[\frac{\Gamma(v)}{\Gamma(u)}\right]^{\frac{y-x}{v-u}} \leq \frac{\Gamma(y)}{\Gamma(x)} \leq\left[\frac{\Gamma(z)}{\Gamma(w)}\right]^{\frac{y-x}{z-w}}
$$

PROOF. Since the function $n \rightarrow \log \Gamma(\eta), n \varepsilon R_{+}$, is convex, it follows from inequality (3) that

$$
\frac{\log \Gamma(v)-\log \Gamma(u)}{v-u} \leq \frac{\log \Gamma(y)-\log \Gamma(x)}{y-x} \leq \frac{\log \Gamma(z)-\log \Gamma(w)}{z-w},
$$

provided $u, v, x, y, w$ and $z$ satisfy the hypothesis of the theorem. Since inequality (5) is equivalent to inequality (4), the proof of the theorem is complete.

COROLLARY 1. For $(\xi, \theta) \in R_{+} \times[0,1]$, we have

$$
(m+\xi)^{1-\theta} \leq \frac{\Gamma(m+\xi+1)}{\Gamma(m+\xi+\theta)} \leq(m+\xi+\theta)^{1-\theta}, m \varepsilon z .
$$


PROOF. Set $u=m+\xi, \dot{v}=m+\xi+1, x=m+\xi+\theta, y=m+\xi+1$, $w=m+\xi+\theta$ and $z=m+\xi+1+\theta$.

Then inequalities (5) reduce to inequalities (6).

The case $\xi=0$ and $0<\theta<1$ is due to Gautschi ([3], 5 3.6.51). Inequalities (6) in the form

$$
\frac{1}{(m+\xi+\theta)^{1-\theta}}<\frac{\Gamma(m+\xi+\theta)}{\Gamma(m+\xi+1)}<\frac{1}{(m+\xi)^{1-\theta}},
$$

were obtained by Lazarević and Lupas [2] who made use of the fact that the Gamma function is logarithmic convex and an unpublished result of Lupas on inequalities involving the Gamma function.

We now prove a more general result which contains, as a special case, an imporved version of Boyd's result [1], namely,

$$
\left\{m+\frac{1}{4}+\frac{1}{32 m+32}\right\}^{\frac{3}{2}}<\frac{\Gamma(m+1)}{\Gamma\left(m+\frac{1}{2}\right)}<\left\{\frac{\left(m+\frac{1}{2}\right)^{2}}{m+\frac{3}{4}+\frac{1}{32 m+32}}\right\}^{\frac{1}{2}} .
$$

We first obtain the following results on differentiable functions:

THEOREM 3. Let $\phi_{1}$ and $\phi_{2}$ be two differentiable real-valued functions on an open interval $S$ in $R$. Let $x, y, u, v \varepsilon S, x \neq y, u \neq w$. Then there exists $\eta \varepsilon(0,1)$ such that for every positive real number $\alpha$,

$$
\begin{aligned}
& \frac{\phi_{1}(y)-\phi_{1}(x)}{y-x}=\frac{\phi_{2}(v)-\phi_{2}(u)}{v-u} \\
& +\alpha \eta^{\alpha-1}\left[\phi_{1}^{\prime}\left(x+\eta^{\alpha}(y-x)\right)-\phi_{2}^{\prime}\left(u+\eta^{\alpha}(v-u)\right)\right] .
\end{aligned}
$$

PROOF. Consider the function

$$
F(\lambda)=\frac{v-u}{\alpha} \phi_{1}\left(x+\lambda^{\alpha}(y-x)\right)-\frac{y-x}{\alpha} \phi_{2}\left(u+\lambda^{\alpha}(w-u) .\right.
$$


This function is differentiable on $[0,1]$. By the usual Mean Value Theorem for differentiable functions, we obtain the desired conclusion.

THEOREM 4. Let $\phi$ be a differentiable real-valued function on an open interval $S$ in $R$ and let $\phi^{\prime}$ be non-decreasing on $S$. Suppose $u, v, x, y \in S, u \neq v, x \neq y$ and either $x>u, v>y$ or $x<u$, $v<y$. Then, for some $\alpha_{0} \varepsilon z_{+}$(the set of positive integers) such that

$$
\left(1-\eta^{\alpha}\right)(x-u)+\eta^{\alpha}(y-v) \geq 0,0<\eta<1, \alpha \geq \alpha_{0} \text {, }
$$

we have

$$
\frac{\phi(y)-\phi(x)}{y-x} \geq \frac{\phi(v)-\phi(u)}{v-u}
$$

We note, however, that inequality (10) is valld if $x \geq u, y \geq v$ and $\alpha$ is an arbitrary positive real number.

PROOF. Let $\phi_{1}=\phi_{2}=\phi$ in Theorem 3. The assumptions on $x, y, u$ and $v$ Imply that $\frac{x-u}{x-u+v-y}$ is an arbitrary real number between 0 and 1 .

Suppose $0<\eta<\frac{x-u}{x-u+v-y}<1$. Then, for all $\alpha \varepsilon z_{+}$, $n^{\alpha}<\frac{x-u}{x-u+v-y}$. If, however, $0<\frac{x-u}{x-u+v-y}<n<1$, there exists $\alpha_{0} \varepsilon z_{+}$such that for all $\alpha \geq \alpha_{0}, \alpha \varepsilon z_{+}, \eta^{\alpha} \leq \frac{x-u}{x-u+v-y}$. Hence, in elther case, $\left(1-\eta^{\alpha}\right)(x-u)+\eta^{\alpha}(y-v) \geq 0$, for all $\alpha \varepsilon z_{+}, \alpha \geq \alpha_{0}$. The conclusion follows by Theorem 3 and the non-decreasing character of $\phi^{\prime}$.

We remark on passing, that inequality (10) is strict unless $\phi$ is a constant or linear function. Furthermore, Inequality (10) is reversed if $\phi$ is non-increasing.

COROLIARY 2. Let $\phi$ be a twice differentiable real-valued convex function on an open interval $S$ in $R$. Let $x, y, u$ and $v$ satisfy the conditions of Theorem 4. Then inequality (10) holds if inequality (9) is valid. The inequality is reversed if $\phi$ is concave. 
PROOF. Since $\phi$ is convex on $S, \phi^{\prime \prime}$ is non-negative on $S$. Hence $\phi^{\prime}$ is non-decreasing on S. If, however, $\phi$ is concave, $\phi^{\prime}$ is non-increasing on $S$. Consequently, the conclusion of the corollary follows from Theorem 4.

An immediate consequence of the above corollary can be obtained by specializing $\phi$. For example, if we take $\phi(\alpha), \alpha \varepsilon R_{+}$, as $\log \Gamma(\alpha)$, then this function satisfies the condition of Corollary 2. Consequently, 1f inequality (9) holds and $x, y, u, v$ satisfy the conditions of Theorem 4, we have

$$
\frac{\Gamma(y)}{\Gamma(x)} \geq\left\{\frac{\Gamma(v)}{\Gamma(u)}\right\}^{\frac{y-x}{v-u}}
$$

For $m \geq-\frac{1}{2}$, let $\gamma \in R-\{0\}$ be such that $n=\frac{m}{\gamma}, 0<n<1$. Put $x=m+\frac{1}{2}, y=m+1, u=m+\theta(m)$ and $v=m+1+\cdot \theta(m)$ where $\frac{1}{4} \leq \theta(m)<\frac{1}{2}$. Since $x-u>0, y-v<0$ and $\frac{1}{4} \leq \theta(m)<\frac{1}{2}$, inequality (11) holds if and only if for some positive integer $\alpha, \frac{1-n^{\alpha}}{n^{\alpha}} \geq \frac{v-y}{x-u} \geq 1$. Hence

$$
(m+\theta(m))^{\frac{1}{2}} \leq \frac{\Gamma(m+1)}{\Gamma\left(m+\frac{1}{2}\right)} \text { if } \frac{1}{4} \leq \theta(m) \leq \frac{1}{2}\left[1-\left(\frac{m}{\gamma}\right)^{\alpha}\right], 0<\left(\frac{m}{\gamma}\right)^{\alpha} \leq \frac{1}{2} \text {. }
$$

Letting $\alpha \rightarrow \infty$, we get

$$
(m+\theta(m))^{\frac{1}{2}} \leq \frac{\Gamma(m+1)}{\Gamma\left(m+\frac{1}{2}\right)} \text { if } \quad \frac{1}{4} \leq \theta(m) \leq \frac{1}{2}
$$

Now write $v=m+1, u=m+\frac{1}{2}, y=m+1+\theta(m)$ and $x=m+\theta(m)$. Then $x-u<0$ and $v-y<0$. Consequently, inequality (11) holds if and only if $\frac{1-\eta^{\alpha}}{n^{\alpha}} \leq 1 \leq \frac{v-y}{x-u}$. Equivalently,

$$
(m+\theta(m))^{\frac{1}{2}} \geq \frac{\Gamma(m+1)}{\Gamma\left(m+\frac{1}{2}\right)},
$$

provided

$$
\frac{1}{4} \leq \theta(\mathrm{m}) \leq \frac{1}{2}\left[1-\left(\frac{\mathrm{m}}{\gamma}\right)^{\alpha}\right], \frac{1}{2} \leq\left(\frac{\mathrm{m}}{\gamma}\right)^{\alpha}<1
$$


a condition which reduces to $\theta(m)=\frac{1}{4}$.

Combining inequalities (12) and (13), we obtain

$$
(m+\theta(m))^{\frac{1}{2}} \leq \frac{\Gamma(m+1)}{\Gamma\left(m+\frac{1}{2}\right)}, \quad \frac{1}{4} \leq \theta(m) \leq \frac{1}{2} .
$$

The converse of this result was obtained by Watson [4], namely, if

$$
\begin{aligned}
& \frac{\Gamma(m+1)}{\Gamma\left(m+\frac{1}{2}\right)}=(m+\theta(m))^{\frac{1}{2}}, \text { then } \frac{1}{4} \leq \theta(m) \leq \frac{1}{2} \quad \text { for } m \geq-\frac{1}{2} \text { and } \\
& \frac{1}{4} \leq \theta(m) \leq \frac{1}{\Pi} \quad \text { for } \quad m \geq 0 .
\end{aligned}
$$

For $m \geq-\frac{1}{2}, \frac{1}{4}<\theta(m) \leq \frac{1}{2}$, we obtain

$$
\frac{\Gamma(m+1)}{\Gamma\left(m+\frac{1}{2}\right)}=\frac{m+\frac{1}{2}}{\left\{\frac{\Gamma\left(m+\frac{1}{2}\right)}{\Gamma(m+1)}\right\}}<\left\{\frac{\left(m+\frac{1}{2}\right)^{2}}{m+\frac{1}{2}+\theta\left(m+\frac{1}{2}\right)}\right\}^{\frac{3}{2}},
$$

Hence, this inequality and Inequality (14) combined yleld

$$
\left.\{m+\theta(m)\}^{\frac{1}{2}}<\frac{\Gamma(m+1)}{\Gamma\left(m+\frac{1}{2}\right)}<\frac{\left(m+\frac{1}{2}\right)^{2}}{m+\frac{1}{2}+\theta\left(m+\frac{1}{2}\right)}\right\}^{\frac{1}{2}}
$$

where $\frac{1}{4}<\theta(m) \leq \frac{1}{2}$.

Taking $\theta(m)=\frac{1}{4}+\frac{1}{32 m+32}, m=1,2, \ldots \ldots$, we obtain inequality (7). on putting $\theta(m)=\frac{1}{4}+\frac{1}{32 m+8+\frac{36}{4 m-3}}$, we obtain an inequality due to Slavić ([5], Inequality (12)).

A result which is better than any one known, except for the formula (15) of Slavić's paper [5] is obtained by putting 


$$
\theta(m)=\frac{1}{4}+\frac{1}{32 m+8+\frac{36}{4 m+5}}
$$

It is our confecture that formula (15) of Slavić's paper [5] can be obtained from our general result, namely inequality (15), by appropriate choice of $\theta=\left[-\frac{1}{2}, \infty\right] \rightarrow\left[\frac{1}{4} ; \frac{1}{2}\right]$.

\section{REFERENCES}

1. Boyd, A. V. Note on the Paper of Uppuluri, Pacific J. Math. 22 (1967) 9-10.

2. Lazarević, I. B. and A. Lupas. Functional Equations for Wallis and Gamma Functions. Univ. Beograd Publ. Elektrotehn. Fak. Ser. Mat. Fiz., 461-497 (1974) 245-251.

3. Mitrinovic, D. S. Analytic Inequalities. Grundlehren der Alath. Wiss., Band 165, Berlin - Heidelberg - New York, 1970, 400 pp.

4. Watson, G. N. A Note on Gamma Functions, Proc. Edinburg Math. Soc. $\underline{2}$ $(1958 / 59) 11$.

5. Slavić, D. V. On Inequality for $\Gamma(x+1) / \Gamma\left(x+\frac{1}{2}\right)$, Univ. Beograd Publ. Elektrotechn Fak. Ser. Mat. Fiz 499, 17-20. 


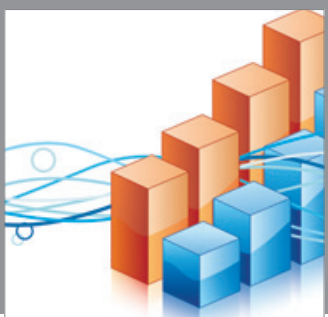

Advances in

Operations Research

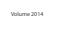

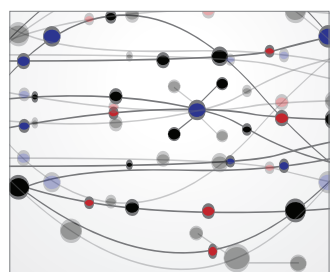

\section{The Scientific} World Journal
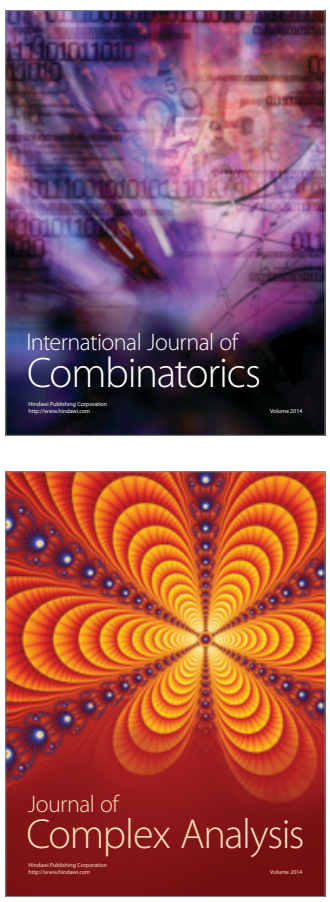

International Journal of

Mathematics and

Mathematical

Sciences
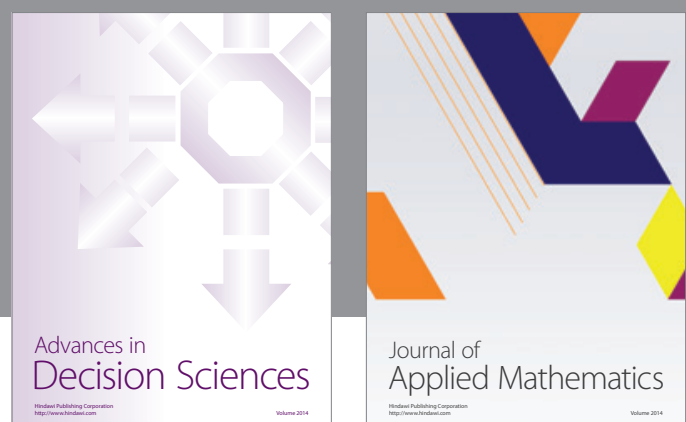

Journal of

Applied Mathematics
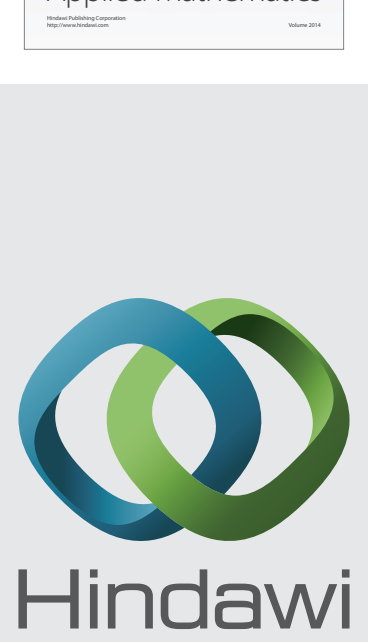

Submit your manuscripts at http://www.hindawi.com
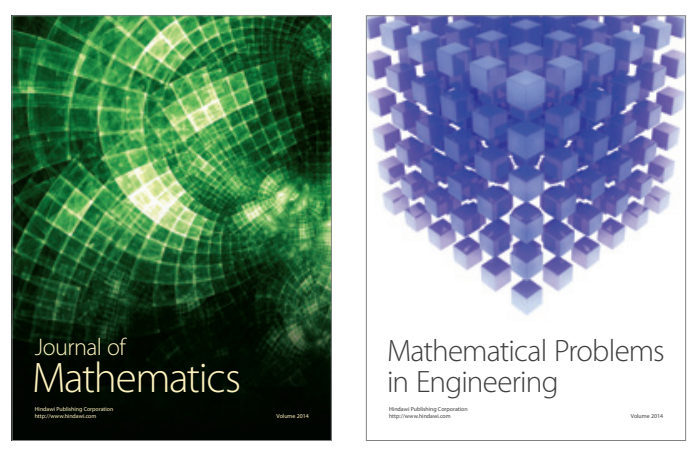

Mathematical Problems in Engineering
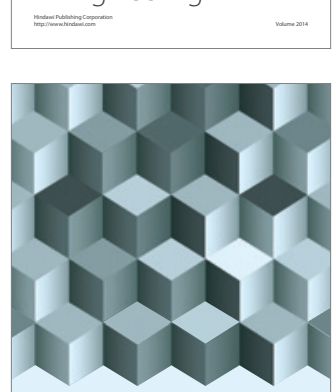

Journal of

Function Spaces
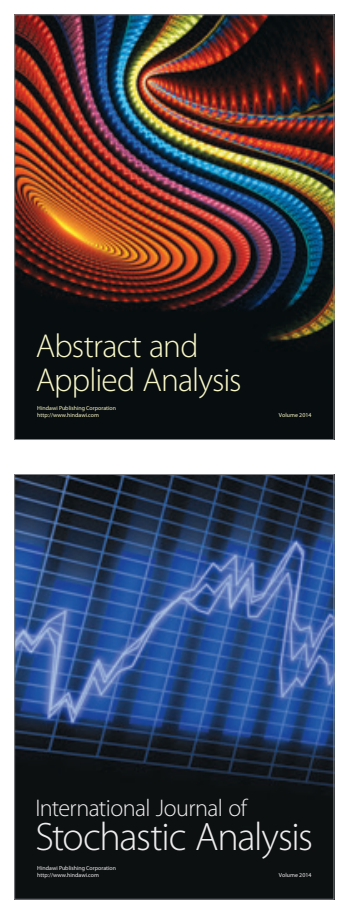

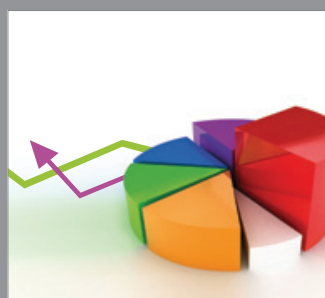

ournal of

Probability and Statistics

Promensencen
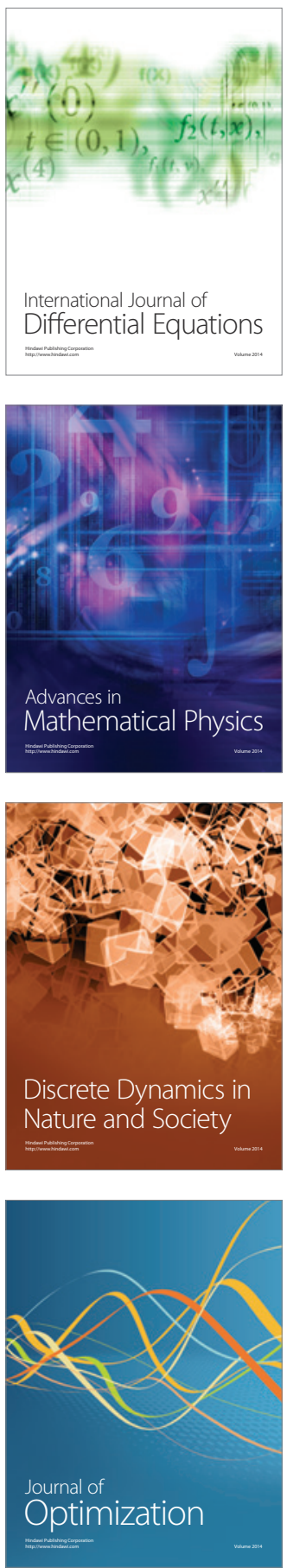